\title{
Fragen zum Thema „Mehr Bewegung bei Diabetes mellitus“
}

\section{- 1. Der Anteil der Bevölkerung, der sich ausreichend bewegt, liegt bei
A $7 \%$.
B $13 \%$.
C $18 \%$.
D $26 \%$.
E $32 \%$.

\section{-2. Welche der folgenden Aussagen trifft zu?}

A Muskelkontraktionen fördern nur unter Insulineinfluss den Glukoseeinstrom in die Muskelzelle.

B Bewegung fördert die Insulinempfindlichkeit durch Steigerung von intramyozellulären Fetteinlagerungen.

C Die Steigerung der Insulinempfindlichkeit durch Bewegung hält ca. vier Tage an.

D Regelmäßige Bewegung führt zu einem nachhaltigen Absinken der Triglyzeridspiegel.

E Um durch Bewegung positive Stoffwechseleffekte zu erzielen, muss es zu einer Gewichtsabnahme kommen.

\section{- 3. Signifikante Verbesserungen für} wesentliche Komponenten des metabolischen Syndroms können bei Menschen mit Diabetes mellitus Typ 2 erwartet werden ab einem Bewegungsumfang (zügiges Gehen) von

A täglich $1 / 2$ Stunde.

B täglich 10 Minuten.

C einmal 1 Stunde pro Woche.

D dreimal 15 Minuten pro Woche.

E täglich 1 Stunde.

- 4. Der ideale Trainingspuls eines 60-jährigen Mannes (60-75\% der maximalen Herzfrequenz) liegt bei

\begin{abstract}
A 100-160 Schlägen pro Minute.
B $80-100$ Schlägen pro Minute.

C 95-125 Schlägen pro Minute.

D 105-145 Schlägen pro Minute.

E 110-150 Schlägen pro Minute.
\end{abstract}

- 5. Die wichtigste Voraussetzung dafür, dass bisher inaktive Menschen mit Diabetes mellitus Typ 2 einen aktiven Lebensstil entwickeln, ist

A das Fehlen von orthopädischen Problemen.

B Motivation durch strukturierte ärztliche Gespräche.

C eine Sportstätte in der Nähe.

D eine gute Sportausrüstung.

E angenehme Witterung.

\section{-6. Welche Aussage trifft zu?}

A In Deutschland sind ca. 3 Mio. Menschen von Typ-2-Diabetes betroffen.

B Durch konsequente ärztliche Beratung behalten zwei Drittel der Patienten über zwei Jahre ihr Trainingsprogramm bei.

C Im Hinblick auf die Fettverbrennung ist Training im anaeroben Bereich sinnvoll.

D Der durchschnittliche Kalorienbedarf eines Erwerbstätigen pro Kopf und Tag liegt derzeit bei etwa $3200 \mathrm{kcal}$.

E Die Compliance ist bei Diäten größer als bei Bewegungsprogrammen.

\section{- 7. Um wie viel kann tägliches zügiges} Gehen für eine halbe Stunde den systolischen Blutdruck durchschnittlich senken?
A - 1,2 mmHg
C $-3,5 \mathrm{mmHg}$
E $-12,2 \mathrm{mmHg}$
B $-2,4 \mathrm{mmHg}$
D $-6,4 \mathrm{mmHg}$

- 8. Wie ist das metabolische Syndrom definiert?

A Übergewicht, Hypertonie, Hyperurikämie, Diabetes mellitus

B Fettleber, Übergewicht, erhöhte Transaminasen, Niereninsuffizienz

C Schlafapnoe-Syndrom, Übergewicht, Polyglobulie, Osteoporose

D Hepatosplenomegalie, Hypertonie, Hypercholesterinämie, Eisenmangel

E Übergewicht, Hypertriglyzeridämie, erniedrigtes HDL-Cholesterin, Hypertonie

\section{- 9. Die endotheliale Dysfunktion}

A ist ein seltenes Phänomen bei Typ-2Diabetes.

B bedeutet eine verminderte Bereitstellung von NO.

C betrifft nicht die koronare Mikrozirkulation.

D wirkt sich über einen NO-Überschuss proatherogen aus.

E kann durch Ausdauertraining kaum positiv beeinflusst werden.

- 10. Um welchen Wert kann das $\mathrm{HbA}_{1 c}$ bei Patienten mit Diabetes mellitus im Lauf von acht Monaten allein durch körperliches Training gesenkt werden?
A $0,12 \%$
B $0,26 \%$
C $0,66 \%$

\section{ANTWORTFORMULAR}

Ich versichere, alle Fragen ohne fremde Hilfe beantwortet zu haben.

Mit dem Einreichen dieses Fragebogens erklärt sich der Einreichende damit einverstanden, dass die angegebenen Daten zum Zweck der Teilnahmebestätigung gespeichert und bei erfolgreicher Teilnahme auch an den Einheitlichen Informationsverteiler (EIV) der Ärztekammern weitergegeben werden.

\section{Wichtiger Hinweis:}

Eine Auswertung ist künftig nur noch möglich, wenn Sie Ihre EFN auf dem Antwortformular angeben! Nähere Hinweise hierzu unter: www.cme-punkt.de/faq.html

„Mehr Bewegung bei Diabetes mellitus“ MMw-Fortschr. Med. Nr. 19 / 2007 (149. Jg.)

$\begin{array}{lllllllllll} & 1 & 2 & 3 & 4 & 5 & 6 & 7 & 8 & 9 & 10\end{array}$

Name, Vorname

Fachrichtung

Geburtsdatum Einheitliche Fortbildungsnummer (EFN)

Und so kommen Sie zu Ihren Punkten:

Teilnahme im Internet: unter www.cme-punkt.de. Dort führen wir für Sie ein elektronisches Punktekonto.

Teilnahme per Brief: Fragebogen ausfüllen und mit einem frankierten Rückumschlag an: Urban \& Vogel Medien und Medizin Verlagsgesellschaft, Redaktion MMW-Fortschritte der Medizin, Abt. Fortbildungszertifikat, Neumarkter Str. 43, 81673 München. 